\title{
588 CALORIC RESTRICTION SENSITIZES MELANOMA TO ANTI-PD-1 IMMUNE CHECKPOINT INHIBITION
}

Alexa Melucci*, Shuyang Qin, Alexander Chacon, Rachel Jewell, Peter Prieto. University of Rochester Medical Center, Rochester, NY, USA

Background Individual response to immune checkpoint inhibition (ICI) in patients with metastatic melanoma varies from $10-40 \%$ for monotherapy and $50-60 \%$ with combination therapy [1]. Identification of adjuncts to ICI to further bridge treatment response to cure is imperative. We focus on caloric restriction (CR) as an adjunct to anti-PD-1 ICI given its inexpensive nature, relative ease of application, and increased tolerability as compared to fasting.

Methods 12-week C57BL/6J mice were inoculated with Yale University Mouse Melanoma (YUMM 1.7), randomized into diet groups and further randomized into $\alpha \mathrm{PD}-1$ and control (IgG) groups on day 12 (D12). Full diet mice ad lib fed while CR mice were $40 \%$ calorically restricted based on average daily food intake. Tumors were measured every 3 days with digital calipers (q3d). $\alpha$ PD-1 or control was intraperitoneally injected starting D12 continuing q3d for 7 injections. Mice were sacrificed and tumors harvested on D31. RNA sequencing was performed on CD45+ CD3 + T cells.

Results Under CR conditions, mice treated with $\alpha \mathrm{PD}-1$ had significantly smaller tumor volumes compared to the full diet cohort treated with $\alpha$ PD-1 (D22, $271.15 \mathrm{~m} 3$ vs. $336.72 \mathrm{~m} 3$, $\mathrm{p}=0.031$ ) and persisted to harvest (D31, $600.96 \mathrm{~m} 3$ vs. $1039.84 \mathrm{~m} 3, \mathrm{p}=0.034)$. A significant difference in tumor volumes between CR $\alpha$ PD- 1 and CR control treated cohorts was observed starting at D28 $(439.34 \mathrm{~m} 3$ vs. $667.63 \mathrm{~m} 3$, $\mathrm{p}=0.005$ ) and persisted to harvest (D31, 600.96 m3 vs. $884.08 \mathrm{~m} 3, \mathrm{p}=0.009$ ). However, no significant difference in tumor growth under full diet conditions in murine cohorts treated with $\alpha \mathrm{PD}-1$ or control or separately between $\mathrm{CR}$ and full diet cohorts treated with control was observed.On pathway enrichment analysis inflammatory response, cytokine-mediated, response to interferon-gamma, and cell proliferation pathways were downregulated in the CR $+\alpha$ PD- 1 cohort. Notable genes found in these pathways include B-cell linker protein (BLNK), tyrosine-protein kinase Lyn (LYN), SYK (spleen tyrosine kinase), toll-like receptor (TLR) TLR4, TLR7, and TLR8.

Conclusions Caloric restriction significantly sensitizes YUMM 1.7 murine melanoma to anti-PD-1 therapy resulting in decreased tumor growth. We show significant modulation of tumor growth in a murine tumor cell line, which has previously demonstrated limited response to $\alpha \mathrm{PD}-1$. In the present study, caloric restriction may decrease inflammation via downregulation of cytokine and toll-like receptor mediated pathways. Furthermore, caloric restriction may reverse the immunosuppressive tumor microenvironment and provide an inexpensive means to increase treatment response to anti-PD-1 therapy.

\section{REFERENCES}

1. Ward WH, Farma JM. Cutaneous Melanoma: Etiology and Therapy. Brisbane (AU): Codon Publications. 2017; Chapter 8.

Ethics Approval This study was approved by the University Committee on Animal Resources (UCAR), UCAR-2018-014.

http://dx.doi.org/10.1136/jitc-2021-SITC2021.588 